\title{
Stabilité des suspensions reconstituées de la combinaison thérapeutique antipaludique à base d'artéméther-luméfantrine avec différents types d'eaux
}

\author{
André Sawa KPAIBE*, François Nicaise BONY, Laeticia Khra YOBOUE, \\ Gildas Komenan GBASSI, Christophe N'cho AMIN, Michèle AKE \\ et Anglade Kla MALAN \\ Département de Chimie Analytique, Bromatologie, Chimie Générale et Minérale, \\ UFR des Sciences Pharmaceutiques et Biologiques, Université Félix Houphouët-Boigny, \\ BPV 34 Abidjan, Cote d'Ivoire. \\ *Auteur correspondant, E-mail : andresawa@yahoo.fr ; BPV 34 Abidjan, Cote d'Ivoire. \\ Tel: $+33753042886,+22540774954$
}

\section{RESUME}

Les combinaisons thérapeutiques antipaludiques à base d'artéméther-luméfantrine sous forme de poudres lyophilisées, sont des préparations multidoses destinées aux enfants pour le traitement du paludisme. Dans le but d'obtenir une bonne observance thérapeutique chez les enfants, ces poudres lyophilisées à reconstituer ont été mises sur le marché. La stabilité des suspensions obtenues après reconstitution avec différents types d'eau accessible à toutes les couches sociales dans les zones endémiques de paludisme a été évaluée. Les caractères organoleptiques et le pH des suspensions ont été recherchés. Les teneurs en artéméther et en luméfantrine ont été recherchés respectivement par chromatographie liquide et par spectrométrie UV. Les produits de dégradation de l'artéméther et de la luméfantrine ont aussi été recherchés par chromatographie sur couche mince de gel de silice. Des études microbiologiques ont été réalisées pour la recherche des germes anaérobies viables totaux et la recherche d'entérobactéries. L'étude a révélé que les caractères organoleptiques et le $\mathrm{pH}$ de chaque suspension ont été acceptables. Les principes actifs n'ont pas été dégradés après reconstitutions des poudres lyophilisées dans tous les échantillons durant la période d'étude. Les concentrations obtenues en artéméther et en luméfantrine de tous les échantillons analysés, étaient conformes aux normes. Les échantillons n'ont pas été contaminés par des bactéries, des levures et des moisissures au cours de cette période d'étude.

(C) 2016 International Formulae Group. All rights reserved.

Mots clés: Stabilité, artéméther, luméfantrine, suspension, spectrophotométrie, chromatographie.

\section{Stability of reconstituted suspension of artemether-luméfantrine lyophilized powders against malaria with different types of water}

\begin{abstract}
The artemether-lumefantrine antimalarial therapeutic combinations in the form of lyophilized powders are multidoses preparations intended for the treatment of children against malaria. For obtaining a good therapeutic observance on the children, the lyophilized powders to be reconstituted were launched on the
\end{abstract}


market. The stability of the suspensions obtained after reconstitution with various types of water accessible to all sectors of society in the endemic zone of malaria was evaluated. The organoleptic characteristics and $\mathrm{pH}$ of the suspensions were evaluated. The concentrations of artemether and lumefantrine were respectively sought by liquid chromatography and UV spectrometry. Also the degradation products of artemether and lumefantrine were searched by thin layer chromatography. Microbiological studies were realized for the search of total viable anaerobic germs and anterobacteria. The study revealed that the organoleptic characteristics and $\mathrm{pH}$ of every suspension were acceptable. Active substances were not degraded after reconstitutions of lyophilized powders in all the samples during the period of study. The artemether and lumefantrine concentrations obtained were in agreement with the standards. Samples were not contaminated by bacteria, yeasts and molds during this period of study.

(C) 2016 International Formulae Group. All rights reserved.

Keywords: Stability, artemether, lumefantrine, , suspension, spectrophotometry, chromatography.

\section{INTRODUCTION}

Le paludisme est une maladie infectieuse causée par les parasites du genre Plasmodium, notamment Plasmodium falciparum, Plasmodium vivax, Plasmodium ovale, Plasmodium malariae, et Plasmodium knowlesi. Il est transmis à l'homme par la piqûre d'un moustique appelé anophèle (WHO, 2014). Le paludisme provoqué par l'infection au Plasmodium falciparum est la principale cause de décès liée au paludisme dans le monde (Bardach et al., 2015). Ce parasite est responsable des formes de paludisme les plus graves chez les enfants (Imbert et al., 2003). Plus de 98 millions de cas de paludisme dont 584.000 décès sont survenus en 2013 dans le monde (WHO, 2014). En Afrique, un enfant décède du paludisme toutes les 20 secondes (Bourée et al., 2006). La prise en charge de cette maladie s'appuie sur l'usage de médicaments antipaludiques tels que les combinaisons thérapeutiques antipaludiques (CTA) dérivées de l'artémisinine. C'est le cas l'artémétherluméfantrine ( $\mathrm{AL})$. Cette CTA est notamment disponible sous forme de poudres lyophilisées à reconstituer. Il s'agit de préparation multidose, mieux adaptées aux enfants chez qui l'observance thérapeutique nécessite des préparations galéniques adéquates (Abdulla et al., 2009; Bassat et al., 2015; Tadesse et al., 2014; Bassat et al., 2014). Elles sont à reconstituer juste au moment de la prise. Après reconstitution, la suspension doit être stable durant le traitement conformément à son dossier d'autorisation de mise sur le marché (Awofisayo et al., 2015). La stabilité constitue un paramètre indispensable à la qualité du médicament (Swartz et al., 2005; Caulin, 2008 ). Cependant, les conditions de reconstitution sont très souvent difficiles à mettre en œuvre dans les zones endémiques à ressources limitées. Certaines couches sociales n'ont pas accès à l'eau minérale de qualité reconnue, qui doit être utilisée pour la reconstitution. Ces populations ont le plus souvent recours à de l'eau dont la qualité n'est pas toujours vérifiée. Or une eau de mauvaise qualité pourrait modifier considérablement la stabilité de ces médicaments et réduire son efficacité. Si la stabilité de ces poudres lyophilisées après reconstitution est acquise avant la mise sur le marché, la variabilité des couches sociales conduirait à s'interroger sur la qualité de l'eau utilisée pour la reconstitution. Il devient donc important d'évaluer la stabilité des suspensions d'AL (Figure 1), reconstituées avec différents types d'eau.

\section{MATERIEL ET METHODES \\ Matériel \\ Appareillages et réactifs}

L'appareillage était composé d'un chromatographe liquide haute performance (Shimadzu, France), d'un spectrophotomètre d'absorption UV-2401 (Shimadzu, France), de plaques de gel de silice 60xFx254 (Merck, 
France), d'un pH-mètre (Mettler Toledo, France), d'une balance (OHAUS, France), d'un bain-marie (STUART, France), d'un agitateur magnétique (Bioblock Scientific, France), d'une centrifugeuse (Herolab, France), d'une plaque chauffante (Stuart, France), du papier-filtre Whatman (grade 3, $70 \mathrm{~mm}$ ). de boîtes de Pétri (Gosselin, France), de pipette Pasteur (Vitlab, Allemangne) et de la verrerie classique de laboratoire.

Les réactifs utilisés tels que l'acétonitrile (Prolabo, France), l'éthanol 96\% (Prolabo, France), le benzène (Prolabo, France), l'acétyle acétate (Prolabo, France) et le n-hexane (Prolabo, France), l'acétone (Sigma-Aldrich, France), diéthylamide (Sigma-Aldrich, France), étaient de qualité analytique. Des solutions pour l'analyse microbiologique ont été utilisées. Il s'agissait de solution peptonée au chlorure de sodium (Merck Millipore, France), de tween 80 (Sigma-Aldrich, France), de liquide peptoné de caséine soja (Becton Dickinson, France), de gélose Sabouraud (Becton Dickinson, France) + Chloramphénicol, de milieu lactosé (Becton Dickinson, France), de milieu liquide de Mossel (Becton Dickinson, France), et de milieu glucosé (Becton Dickinson, France). Les substances de références utilisées pour l'étude étaient la poudre d'artéméther (SigmaAldrich, France) et la poudre luméfantrine (Sigma-Aldrich, France).

\section{Échantillons}

L'étude a porté sur seize (16) flacons de $60 \mathrm{ml}$ de poudre lyophilisée d'AL à reconstituer $(180 \mathrm{mg}$ d'artéméther et $1080 \mathrm{mg}$ de luméfantrine). L'échantillonnage était constitué de huit (8) flacons d'un médicament M1 d'AL (lot 22079) et huit (8) flacons d'un médicament M2 d'AL (lot LU35). Ces échantillons ont été enregistrés en Côte d'Ivoire. Les différents types d'eaux utilisées pour la reconstitution étaient composés de deux (2) marques d'eau minérale embouteillée commercialisées en Côte d'Ivoire (EM 1 et EM 2), de l'eau d'adduction provenant du robinet (ER) et de l'eau de robinet bouillie refroidie (ERB).

\section{Méthodes \\ Préparation des échantillons et solutions étalons}

Cinq milligrammes de poudre d'artéméther et $1 \mathrm{mg}$ de poudre de luméfantrine ont été chacun dissous avec de l'éthanol 96\% dans une fiole jaugée de $10 \mathrm{ml}$, puis filtré sur du papier-filtre Whatman. Différentes dilutions ont ensuite été effectuées. D'abord à partir de la solution mère d'artéméther avec le mélange acétonitrile/eau (1/1) pour obtenir des solutions de concentration comprises entre $960 \mu \mathrm{g} / \mathrm{ml}$ et $1440 \mu \mathrm{g} / \mathrm{ml}$. Ensuite à partir de la solution mère de luméfantrine pour obtenir des solutions de concentrations comprises entre $29,1 \mu \mathrm{g} / \mathrm{ml}$ et $43 \mu \mathrm{g} / \mathrm{ml}$. Les poudres lyophilisées d'AL (180 mg/1080 mg) ont été reconstitués en ajoutant $60 \mathrm{ml}$ d'eau dans les flacons contenant le médicament le premier jour des analyses (J0). Chaque type d'eau a été utilisé pour la reconstitution de 2 médicaments (M1 et M2).

\section{Évaluation de la dégradation des solutions}

Les paramètres organoleptiques tels que l'aspect, la couleur, la saveur et l'odeur, ainsi que le $\mathrm{pH}$ des suspensions reconstituées ont été évalué sur 14 jours de conservation (J0, J3, J7 et J14). La recherche des produits de dégradation a fait appel à des méthodes tirées de la pharmacopée internationale $\left(4{ }^{\text {ème }}\right.$ édition). Les produits de dégradation ont été recherchés à $\mathrm{J} 0, \mathrm{~J} 3, \mathrm{~J} 7$ et $\mathrm{J} 14$ par chromatographie sur couche mince de gel de silice. La recherche de produits de dégradation de l'artéméther a utilisé une phase mobile constituée du mélange benzène/acétate d'éthyle (90/10) et une révélation à la vapeur d'iode. La recherche de produits de dégradation de la luméfantrine a utilisé une phase mobile constituée du mélange nhexane/acétone/diéthylamine (80/14/6) et une révélation à la lumière UV à $254 \mathrm{~nm}$.

\section{Dosage de l'artéméther et de la luméfantrine}

Deux millilitres de chaque suspension reconstituée d'AL ont été introduit dans des fioles jaugées de $10 \mathrm{ml}$, puis $5 \mathrm{ml}$ d'éthanol ont été ajoutés. L'ensemble a été mélangé à 
l'aide d'un agitateur magnétique (800 rpm) pendant 20 minutes. Le volume a été complété jusqu'au trait de jauge avec le même solvant, puis filtré sur du papier-filtre Whatman. L'identification et le dosage de l'artéméther et de la luméfantrine ont fait appel à des méthodes tirées de la pharmacopée internationale (4 ${ }^{\text {ème }}$ édition). La teneur en artéméther a été déterminée par chromatographie liquide sur une colonne C18. La phase mobile était constituée du mélange acétonitrile/eau (1/1) pour un débit de $2 \mathrm{ml} /$ min. La détection UV était fixée à $210 \mathrm{~nm}$. La luméfantrine a été quantifiée par spectrophotométrie UV-visible à $339 \mathrm{~nm}$.

\section{Procédure de validation analytique}

Des paramètres ont été évalués pour apprécier la fiabilité des résultats obtenus ont été évalué selon la procédure $\mathrm{ICH}$ Harmonised Tripartite (2005). L'étude de la linéarité des teneurs en artéméther et en luméfantrine a été réalisée sur des produits de référence. Elle a porté sur des concentrations successives en artéméther de $960 \mu \mathrm{g} / \mathrm{ml}, 1080$ $\mu \mathrm{g} / \mathrm{ml}, 1200 \mu \mathrm{g} / \mathrm{ml}, 1320 \mu \mathrm{g} / \mathrm{ml}$, et 1440 $\mu \mathrm{g} / \mathrm{ml}$; puis sur des concentrations successives en luméfantrine de $29,1 \mu \mathrm{g} / \mathrm{ml}, 32$ $\mu \mathrm{g} / \mathrm{ml}, 36 \mu \mathrm{g} / \mathrm{ml}, 39,2 \mu \mathrm{g} / \mathrm{ml}$, et $43 \mu \mathrm{g} / \mathrm{ml}$. La répétabilité $(n=6)$ a été établie sur des concentrations successives des solutions de référence d'artéméther $(1080 \mu \mathrm{g} / \mathrm{ml}, 1200$ $\mu \mathrm{g} / \mathrm{ml}, 1440 \mu \mathrm{g} / \mathrm{ml})$ et de luméfantrine $(29,1$ $\mu \mathrm{g} / \mathrm{ml}, 32 \mu \mathrm{g} / \mathrm{ml}, 39,2 \mu \mathrm{g} / \mathrm{ml})$. L'exactitude a été déterminée par la méthode des ajouts dosés à partir des solutions de références d'artéméther $(960 \mu \mathrm{g} / \mathrm{ml})$ et de luméfantrine $(29,1 \mu \mathrm{g} / \mathrm{ml})$. Les limites de détection et de quantification ont été évaluées à partir de dilutions successives de solutions de références d'artéméther et de luméfantrine.

\section{Recherche de contaminants}

Tous les tests microbiologiques ont été réalisés dans des conditions stériles.

Germes aérobies viables totaux: Dix millilitres de chaque échantillon d'AL a été introduit dans une fiole conique stérile de 250 $\mathrm{ml} .100 \mathrm{ml}$ de solution peptonée au chlorure de sodium à $0,1 \%$ de tween 80 stérile, a été ajouté lentement. L'ensemble a été mélangé sur l'agitateur magnétique (800 rpm) pendant 10 minutes pour obtenir la solution «mère». Ensuite cette solution «mère»a été diluée au $1 / 10^{\text {ème }}$ dans une autre fiole conique stérile de $100 \mathrm{ml}$ avec la même solution pour obtenir une solution «fille». Les solutions «mère»et «fille» obtenues ont été portées au bain-marie à $40{ }^{\circ} \mathrm{C}$, pendant 30 minutes, puis mélangées sur l'agitateur magnétique (800 rpm) jusqu'à homogénéisation. Un millilitre de solution «mère» et $1 \mathrm{ml}$ de solution «fille» ont été déposé respectivement dans 2 boîtes de pétri pour chaque solution, à l'aide d'une pipette pasteur stérile. Pour le dénombrement des bactéries, le milieu liquide peptoné de caséine soja stérile a été coulé dans les boîtes de pétri. Les boîtes de pétri ont ensuite été mises à l'étuve à $37^{\circ} \mathrm{C}$, pendant 24 heures (Adeshina et al. 2009).

Les colonies (n) de bactéries ont ensuite été dénombrées. Pour le dénombrement des levures et des moisissures, le milieu gélose Sabouraud-Chloramphénicol stérile a été coulé, dans les mêmes boîtes de Pétri. Puis ces boîtes de pétri ont été maintenues à la température de $25^{\circ} \mathrm{C}$, pendant 5 jours. Le nombre de colonies a été déterminé dans les mêmes conditions. Le nombre de colonie par millilitre de prise d'essai $(\mathrm{N})$ est déterminé pour une norme fixée à 1000 UFC/ml (Lund, 1994 ; Doukan 2015).

Entérobactéries: Dix millilitre de chaque échantillon d'AL a été introduit dans une fiole stérile de $500 \mathrm{ml} .100 \mathrm{ml}$ de milieu lactosé a ensuite été ajouté dans la fiole. Le contenu a été mélangé manuellement par retournement jusqu'à l'obtention d'une solution homogène. Cette solution a été mise à incuber à l'étuve à $37{ }^{\circ} \mathrm{C}$ pendant 5 heures. Ensuite $10 \mathrm{ml} \mathrm{du}$ milieu préparé a été introduit dans une seconde fiole stérile de $500 \mathrm{ml}$. Puis $100 \mathrm{ml}$ de milieu liquide de Mossel a été ajouté. L'ensemble a été mélangé manuellement par retournement jusqu'à l'obtention d'une solution homogène et a été mis à incuber à l'étuve à 37 ${ }^{\circ} \mathrm{C}$ pendant 48 heures. Le milieu obtenu a été ensemencé en surface et en stries, dans 2 boîtes de Pétri contenant un milieu glucosé au 
cristal violet, au rouge neutre et à la bile. Ensuite les boîtes de Pétri ont été ensuite mises à incuber à l'étuve à $37{ }^{\circ} \mathrm{C}$ pendant 24 heures. Le nombre d'UFC a été déterminé (Lund, 1994 ; Centre d'expertise en analyse environnementale du Québec 2013).

\section{RESULTATS}

L'étude des caractères organoleptiques des suspensions obtenues après reconstitution des poudres lyophilisées d'artéméther et de luméfantrine (Tableau 1) n'a montré aucun signe de non-conformité. Les $\mathrm{pH}$ des eaux utilisées pour la reconstitution des poudres lyophilisées d'AL ont été de 7,1 pour EM1 et ERB; de 6,5 pour ER et de 5,1 pour EM2 . Les $\mathrm{pH}$ obtenus des poudres lyophilisées d'AL reconstituées avec différents type d'eau (Tableau 2) ont révélé que toutes les suspensions obtenues ont un caractère acide. La recherche des produits de dégradation dans les suspensions reconstituées n'a pas révélé de tache caractérisant ces substances sur les plaques CCM.

La validation de la méthode chromatographique appliquée à l'analyse d'une solution de référence d'artéméther (960 $\mu \mathrm{g} / \mathrm{ml})$ a rapporté sur le chromatogramme obtenu, le pic de l'artéméther à 9 minutes (Figure 2). Le domaine de linéarité a été mis en évidence pour des concentrations en artéméther comprises entre 0,96 et $1,44 \mathrm{mg} / \mathrm{ml}$ avec un coefficient de détermination de 0,997 et une droite de régression d'équation Y=98753X-14502. L'étude de la répétabilité sur les concentrations successives de la solution de référence d'artéméther a révélé des coefficients de variation de $0,59 \%, 0,59 \%$ et $0,29 \%$. Le pourcentage moyen de récupération exprimant l'exactitude a été de $99,96 \%$. Les limites de détection et de quantification obtenues ont été respectivement de $2,50 \mu \mathrm{g} / \mathrm{ml}$ et de $76,8 \mu \mathrm{g} / \mathrm{ml}$. La validation de la méthode appliquée à l'analyse de la luméfantrine a rapporté un domaine de linéarité pour des concentrations comprises entre 29,1 et $43 \mu \mathrm{g} / \mathrm{ml}$ avec un coefficient de détermination de 0,996 et une droite de régression d'équation $\mathrm{Y}=14,84 \mathrm{X}-0,005$. L'étude de la répétabilité sur les concentrations successives de la solution de référence de luméfantrine a révélé des coefficients de variation de $0,60 \%, 0,26 \%$ et $0,09 \%$. Le pourcentage moyen de récupération obtenu exprimant l'exactitude a été de 99,99\%. Les limites de détection et de quantification obtenues étaient respectivement de $0,97 \mu \mathrm{g} / \mathrm{ml}$ et de $5,82 \mu \mathrm{g} / \mathrm{ml}$. Les dosages de l'artéméther et de la luméfantrine dans les échantillons de médicaments (M1 et M2) préparés avec les différents types d'eaux, ont rapporté des concentrations en artémether (Tableau 3) et en luméfantrine (Tableau 4) comprises dans l'intervalle de conformité de l'artéméther, [162-198] $\mathrm{mg} / 60 \mathrm{ml}$ et de la luméfantrine [972-1188] $\mathrm{mg} / 60 \mathrm{ml}$. Les coefficients de variation obtenus sur 14 jours de conservation après reconstitutions des poudres lyophilisées ont été inférieurs à $2 \%$ (Valeur de référence).

L'analyse microbiologique des échantillons de poudres lyophilisées reconstituées n'a pas révélé de contamination par des bactéries, des levures et des moisissures au cours des manipulations des flacons de médicaments sur une période de 14 jours.

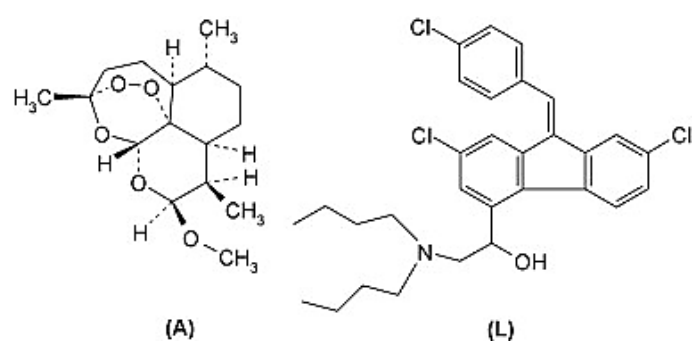

Figure 1 : Structures chimiques de l'artéméther (A) et de la luméfantrine (L). 


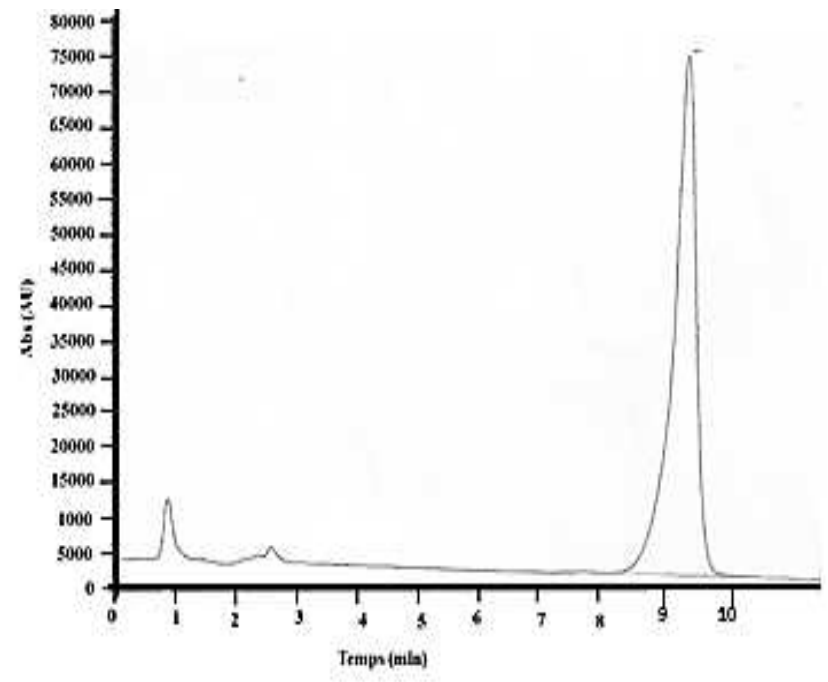

Figure 2: Chromatogramme du dosage de la solution de référence de l'artéméther exprimant l'absorbance (Abs) en fonction du temps en minute (min).

Tableau 1 : Caractères organoleptiques des médicaments M1 et M2.

\begin{tabular}{ccc}
\hline & M1 & M2 \\
\hline Aspect & Liquide visqueux & Liquide visqueux \\
Couleur & Jaune citron & Orange \\
Saveur & Bon goût & Bon goût \\
Odeur & Agréable & Agréable \\
\hline
\end{tabular}

Tableau 2 : pH des poudres lyophilisée reconstituées des médicaments M1 et M2.

\begin{tabular}{cccc}
\hline Jours & Type d'eau & M1 & M2 \\
\hline \multirow{3}{*}{ J0 } & EM 1 & 4,26 & 5,12 \\
& EM 2 & 3,51 & 4,88 \\
& ERB & 3,62 & 5,04 \\
& ER & 3,85 & 4,9 \\
\hline \multirow{3}{*}{ 33 } & EM 1 & 4,18 & 5,11 \\
& EM 2 & 3,5 & 4,89 \\
& ERB & 3,59 & 5,03 \\
J7 & ER & 3,88 & 4,92 \\
& EM 1 & 4,29 & 5,11 \\
& EM 2 & 3,59 & 4,88 \\
& ERB & 3,69 & 5,01 \\
J14 & ER & 3,9 & 5 \\
\hline \multirow{3}{*}{} & EM 1 & 4,22 & 5,1 \\
& EM 2 & 3,55 & 4,86 \\
& ERB & 3,57 & 5,02 \\
& ER & 3,88 & 4,93
\end{tabular}

EM1 : eau minérale de la marque 1; EM2 : eau minérale de la marque2; ER: eau du robinet; ERB : eau du robinet bouillie. 
A. S. KPAIBE et al. / Int. J. Biol. Chem. Sci. 10(2): 894-903, 2016

Tableau 3 : Résultats du dosage de l'artéméther et de la luméfantrine dans le médicament M1.

\begin{tabular}{|c|c|c|c|c|c|c|c|c|c|}
\hline \multirow{2}{*}{$\begin{array}{l}\text { Molécules } \\
\text { Type d'eau }\end{array}$} & & \multicolumn{4}{|c|}{ Artéméther } & \multicolumn{4}{|c|}{ Luméfantrine } \\
\hline & & EM1 & EM2 & ER & ERB & EM1 & EM2 & ER & ERB \\
\hline \multicolumn{2}{|c|}{$\begin{array}{l}\text { Concentration théorique } \\
(\mathrm{mg} / 60 \mathrm{ml}\end{array}$} & \multicolumn{4}{|c|}{180} & \multicolumn{4}{|c|}{1080} \\
\hline Concentration & J0 & 168,884 & 168,8071 & 168,0425 & 169,4925 & 1060,31 & 1052,224 & 1055,2565 & 1059,299 \\
\hline mesurée & $\mathrm{J} 3$ & 171,193 & 168,753 & 169,47 & 169,644 & 1049,1915 & 1042,116 & 1041,105 & 1045,148 \\
\hline \multirow[t]{2}{*}{$(\mathrm{mg} / 60 \mathrm{ml})$} & $\mathrm{J} 7$ & 169,192 & 169,175 & 168,3745 & 168,9675 & 1033,0185 & 1022,551 & 1033,0185 & 1036,051 \\
\hline & $\mathrm{J} 14$ & 170,174 & 169,1905 & 168,951 & 168,8725 & 1023,922 & 1014,8245 & 1037,946 & 1026,9545 \\
\hline Moyenne & & 169,861 & 169,981 & 168,709 & 169,244 & 1041,611 & 1036,304 & 1041,863 & 1040,852 \\
\hline $\mathrm{CV} \%$ & & 0,70 & 0,44 & 1,02 & 0,36 & 1,46 & 1,46 & 1,30 & 0,93 \\
\hline
\end{tabular}

$\mathrm{EM} 1=$ eau minérale de la marque $1 ; \mathrm{EM} 2=$ eau minérale de la marque $2 ; \mathrm{ER}=$ eau du robinet $; \mathrm{ERB}=$ eau du robinet bouillie.

Tableau 4 : Résultats du dosage de l'artémether et de la luméfantrine dans le médicament M2.

\begin{tabular}{llcccccccc}
\hline Molécules & \multicolumn{4}{c}{ Artéméther } & \multicolumn{2}{c}{ Luméfantrine } \\
\hline Type d'eau & & EM1 & EM2 & ER & ERB & EM1 & EM2 & ER & ERB \\
Concentration théorique (mg/60ml & & & \multicolumn{2}{c}{180} & & & & 1080 & \\
\hline Concentration mesurée (mg/60ml) & J0 & 173,268 & 173,026 & 171,982 & 170,928 & 1044,1375 & 1040,0945 & 1036,051 & 1033,0185 \\
& J3 & 171,8175 & 174,9375 & 173,0095 & 173,242 & 1038,0725 & 1033,0185 & 1041,105 & 1032,013 \\
& $\mathrm{~J} 7$ & 173.8605 & 173,1575 & 171,72 & 172,4505 & 1025,9435 & 1020,889 & 1019,8785 & 1017,857 \\
& $\mathrm{~J} 14$ & 171,971 & 173.2675 & 172.9695 & 173,5345 & 1021,9005 & 1020,8895 & 1016,846 & 1026,954 \\
\hline Moyenne & & 172,793 & 173,597 & 172,421 & 172,540 & 1032,513 & 1028,723 & 1028,470 & 1027,460 \\
CV \% & & 0,61 & 0,68 & 0,51 & 0,68 & 0,96 & 0,89 & 1,13 & 0,72 \\
\hline
\end{tabular}

EM1 = eau minérale de la marque $1 ; \mathrm{EM} 2=$ eau minérale de la marque2 $; \mathrm{ER}=$ eau du robinet $; \mathrm{ERB}=$ eau du robinet bouillie. 


\section{DISCUSSION}

L'étude des caractères organoleptiques a révélé que l'aspect, la couleur, le goût et l'odeur des poudres lyophilisées reconstituées d'AL n'ont pas été modifié dans l'ensemble des échantillons analysés. De plus, le pH de chaque échantillon a été stable durant 14 jours de conservation des flacons à température ambiante. L'étude en chromatographie sur couche mince de ces suspensions reconstituées a révélé des taches caractéristiques de l'artéméther et de la luméfantrine à J0. Elle n'a cependant pas révélé de substances de dégradation de ces principes actifs dans tous les échantillons de suspensions reconstituées durant 14 jours de conservation. Ces résultats peuvent justifier la non-dégradation des suspensions reconstituées durant cette période. Cependant, pour tous les échantillons, les poudres lyophilisées ont été difficiles à reconstituer en solution. Ces difficultés pourraient être liées à l'humidité due aux conditions de conservation des flacons.

Concernant la validation des méthodes d'analyse chromatographique et spectrométrique, les résultats des critères de validation que sont la linéarité, la précision et l'exactitude sont apparus satisfaisants conformément aux normes ICH Harmonised Tripartite (2015).

Le chromatogramme obtenu à l'issu de l'analyse de la solution de référence de l'artéméther a rapporté le pic de l'artéméther à 9 minutes (Figure 2) conformément aux travaux de Da Costa et al. (2008). Les domaines de linéarité mis en évidence pour des concentrations en artéméther comprises entre 0,96 et $1,44 \mathrm{mg} / \mathrm{ml}$ et en luméfantrine comprises entre 29,1 et $43 \mu \mathrm{g} / \mathrm{ml}$ ont été significatifs. L'étude de la répétabilité a révélé des coefficients de variation pour la solution de référence d'artéméther et de luméfantrine inférieurs à la norme (2\%). Les pourcentages moyens de récupération de l'artéméther et de la luméfantrine exprimant l'exactitude ont été conformes à la norme comprise entre $90 \%$ et $110 \%$. Ces méthodes peuvent être appliquées aux dosages des teneurs en artéméther et en luméfantrine dans les suspensions reconstituées d'AL. En ce qui concerne l'analyse des échantillons de poudres lyophilisées reconstituées d'AL, les concentrations d'artéméther et de luméfantrine obtenues pour tous les échantillons étaient conformes aux normes. Ces résultats permettent d'attester de la stabilité chimique des suspensions obtenues après reconstitution.

En ce qui concerne les études microbiologiques effectuées, tous les échantillons analysés ont révélé que les poudres reconstituées n'ont pas été contaminées par des bactéries, des levures et des moisissures. En effet, les excipients utilisés pour la fabrication de ces poudres lyophilisées (l'acide citrique, le 4hydroxybenzoate de méthyle, le 4hydroxybenzoate de propyle) sont connus pour leurs activités conservatrices, antibactériennes et antifongiques. (Ryssel $\mathrm{H}$. 2009, Kosova, 2015). Les poudres lyophilisées reconstituées d'AL, se sont donc révélées stables sur une période de 14 jours à $25^{\circ} \mathrm{C}$. Ce résultat est similaire à celui obtenu lors de l'étude de stabilité des suspensions reconstituées à base de cette association sur 30 jours à $30^{\circ} \mathrm{C}$ (Herwig, 2007).

Dans des zones endémiques de paludisme comme en Côte d'Ivoire, les couches sociales défavorisées, qui n'ont pas accès à l'eau minérale (recommandée) pour reconstituer ces médicaments pourront utiliser l'eau potable d'adduction provenant du robinet. L'eau du robinet potable s'étant révélée bien adaptée pour la préparation de poudres lyophilisées antipaludiques d'AL.

\section{Conclusion}

La présente étude a proposé d'évaluer la stabilité des poudres lyophilisées d'AL à reconstituer utilisée dans le traitement du paludisme chez l'enfant. Les analyses physicochimiques réalisées ont révélé que ces suspensions présentaient des caractères organoleptiques acceptables. Les 
concentrations d'artéméther et de luméfantrine obtenues après reconstitution par différents types d'eau (l'eau minérale et l'eau de robinet) étaient conformes aux indications $\mathrm{du}$ fabricant. Les études microbiologiques n'ont pas révélé de contamination sur toute la période de conservation. Le contrôle physicochimique et microbiologique des poudres lyophilisées reconstituées d'AL par de l'eau potable provenant du robinet a montré une bonne stabilité de ces médicaments.

\section{CONFLIT D'INTERET}

Les auteurs déclarent ne pas avoir de conflits d'intérêts en relation avec cet article.

\section{CONTRIBUTIONS DES AUTEURS}

ASK a contribué à l'exploitation des résultats et à la rédaction du manuscrit. KLY et FNB ont contribué à la réalisation des dosages et de l'étude microbiologique. GKB et CNA ont contribué à la correction du manuscrit. MA et AKM ont initié et supervisé les travaux.

\section{REFERENCES}

Abdulla S, Sagara I. 2009. Dispersible formulation of artemether/lumefantrine: specifically developed for infants and young children. Malaria Journal, 8(1): S7.

Adeshina GO, Ajayi S, Onaolapo JA. 2009. Microbiological quality of some commercially available paediatric antimalarial and cough preparations in Ilorin, Nigeria. Nigerian Journal of Pharmaceutical Sciences, 8(1): 109-117.

Adjei GO, Goka BQ, Binka F, Kurtzhals, JA. 2009. Artemether-lumefantrine: an oral antimalarial for uncomplicated malaria in children. Expert Review of Antiinfective Therapy, 7(6): 669-681.

Awofisayo SO, Okhamafe AO, Arhewoh MI. 2015. Determination of elemental impurities in some commercial paediatric and adult formulations of artemether-lumefantrine in the Nigerian market by atomic absorption spectroscopy. Indonesian Journal of Pharmacy, 25(3): 171.

Baird RM, Hodges NA, Denyer SP. 2003. Handbook of Microbiological Quality Control in Pharmaceuticals and Medical Devices: CRC Press.

Bardach A, Ciapponi A, Rey-Ares L, Rojas J I, Mazzoni A, Glujovsky D, Boulos M. 2015. Epidemiology of Malaria in Latin America and the Caribbean from 1990 to 2009: Systematic Review and MetaAnalysis. Value in Health Regional Issues, 8:69-79.

Bassat Q, Ogutu B. DJimde A, Stricker K, Hamed K. 2014. Development of a pediatric formulation for treatment of $P$. falciparum malaria: $\quad$ Coartem ${ }^{\circledR}$ (artemether-lumefantrine) Dispersible. Malaria Journal, 13(1):1-1.

Bassat Q, Ogutu B, Djimde A, Stricker K, Hamed K. 2015. Tailoring a Pediatric Formulation of ArtemetherLumefantrine for Treatment of Plasmodium falciparum Malaria. Antimicrobial Agents and Chemotherapy, 59(8): 4366-4374.

Bourée P. 2006. Aspects actuels du paludisme. Revue Francophone des Laboratoires, 6(385) : 25-38

Caulin C. 2008. Historique de l'évaluation des médicaments en vue d'une autorisation de mise sur le marché. Journal Français d'Ophtalmologie, 31(1): 71-74.

Centre d'expertise analytique du Quebec. 2013. Recherche et dénombrement d'Escherichia coli thermotolérants dans les échantillons solides ou semi-solide : méthode par filtration sur menbrane utilisant le milieu de culture mFC-BCIG, MA. 705 - Ec-BCIG 1.0, Ministère du Développement durable, de l'Environnement, de la Faune et des Parcs du Québec.

Da Costa CI, Nogueira FHA, Pianetti GA. 2008. Simultaneous determination of artemether and lumefantrine in fixed dose combination tablets by HPLC with 
UV detection. Journal of Pharmaceutical and Biomedical analysis, 48(3): 951-954.

Doukan K. 2015. Etude comparative entre le couscous industriel et le couscous à base de glands. Nature and Technology, 13: 2.

Herwig J. 2007. Stability of the Artemether/lumefantrine suspension. Archives WHO int.

ICH Harmonised Tripartite. 2005. Validation of analytical procedures: text and methodology. ICH Expert Working Group.

Imbert P. 2003. Paludisme de l'enfant : critères de gravité. Archives de Pédiatrie, 10: 532-538.

Kosova M, Hradkova I, Matlova V, Kadlec D, Smidrkal J, Filip V. 2015. Antimicrobial effect of $4 \square$ hydroxybenzoic acid ester with glycerol. Journal of Clinical Pharmacy and Therapeutics, 40(4):436440.

Lund W. 1994. The Pharmaceutical Codex, Principle and Practice of Pharmaceutics. Pharmaceutical Press, London; 199: 987-992.

Ogunyemi AK, Buraimoh OM, Onuora NO, Ezeugwu SMC, Odetunde SK,
Olumuyiwa EO. 2015. Bacteria associated with contamination of readyto-eat (RTE) cooked rice in LagosNigeria. Int. J. Biol. Chem. Sci., 9(5), 2324-2333.

Ryssel H, Kloeters O, Germann G, Schäfer T, Wiedemann G, Oehlbauer M. 2009. The antimicrobial effect of acetic acid. An alternative to common local antiseptics? Burns, 35(5): 695-700.

Swartz M, Krull I. 2005. Developing and validating stability-indicating methods. LC GC North America, 23(6): 586-593.

Tadesse AN, Eshetu EM. 2014. Artemetherlumefantrine: Pediatric formulations for the treatment of uncomplicated Plasmodium falciparum. Journal of Scientific and Innovative Research, 3(1): 102-111.

WHO. 2014. World Malaria Report 2014. WHO Press 2014; Geneva.

WHO. 2011. Pharmacopée Internationale ( $4^{\text {ème }}$ édn). WHO: Geneva. 